\title{
A!
}

This is an electronic reprint of the original article.

This reprint may differ from the original in pagination and typographic detail.

Tariq, Usama; Muthusamy, Rajkumar; Kyrki, Ville

\section{Grasp Planning for Load Sharing in Collaborative Manipulation}

\section{Published in:}

Proceedings of the 2018 IEEE International Conference on Robotics and Automation, ICRA 2018

DOI:

10.1109/ICRA.2018.8460579

Published: 01/01/2018

Document Version

Peer reviewed version

Please cite the original version:

Tariq, U., Muthusamy, R., \& Kyrki, V. (2018). Grasp Planning for Load Sharing in Collaborative Manipulation. In Proceedings of the 2018 IEEE International Conference on Robotics and Automation, ICRA 2018 (pp. 68476854). (IEEE International Conference on Robotics and Automation ICRA). IEEE.

https://doi.org/10.1109/ICRA.2018.8460579

This material is protected by copyright and other intellectual property rights, and duplication or sale of all or part of any of the repository collections is not permitted, except that material may be duplicated by you for your research use or educational purposes in electronic or print form. You must obtain permission for any other use. Electronic or print copies may not be offered, whether for sale or otherwise to anyone who is not an authorised user. 
This is the accepted version of the original article published by IEEE.

(C) 2018 IEEE. Personal use of this material is permitted. Permission from IEEE must be obtained for all other uses, in any current or future media, including reprinting/republishing this material for advertising or promotional purposes, creating new collective works, for resale or redistribution to servers or lists, or reuse of any copyrighted component of this work in other works. 


\title{
Grasp Planning for Load Sharing in Collaborative Manipulation
}

\author{
Usama Tariq, Rajkumar Muthusamy and Ville Kyrki
}

\begin{abstract}
In near future, robots are envisioned to work alongside humans in unstructured professional and domestic environments. In such setups, collaborative manipulation is a fundamental skill that allows manipulation of heavy loads by load sharing between agents. Grasp planning plays a pivotal role for load sharing but it has not received attention in the literature. This work proposes a grasp analysis approach for collaborative manipulation that allows load sharing by minimizing exerted grasp wrenches in a task specific way. The manipulation task is defined as expected external wrenches acting on the target object. The analysis approach is demonstrated in a two-agent decentralized set-up with unknown objects. After the first agent has grasped the target, the second agent observes the first agent's grasp location and plans its own grasp according to optimal load sharing. The method was verified in a human robot collaborative lifting task. Experiments with multiple objects show that the proposed method results in optimal load sharing despite limited information and partial observability.
\end{abstract}

\section{INTRODUCTION}

Cooperative ${ }^{1}$ manipulation of large and heavy objects has become an important task in several robotic application domains such as construction, forestry, agriculture and service robotics [1], [2]. To perform such tasks, multiple agents need to coordinate to grasp an object such that a closed kinematic chain is formed. This setting increases the handling and task capacity of individual agents and improves the task precision. Effective coordination can improve the distribution of load among the cooperative agents. Most studies [3], [4], [5] consider multi-agent coordination in centralized setting requiring central decisions from global planning. In contrast, coordination of decentralized systems [6] is more challenging due to the limitations in communication, heterogeneity of agents and incomplete knowledge.

In human-centred robot applications [7], a robot is expected to collaborate with humans and accomplish manipulation tasks together. Such systems are then inherently decentralized and heterogeneous. Moreover, the robot may lack information about the target object and the cooperating human which makes the use of traditional wrench space quality measures inapplicable for grasp planning.

The load sharing problem [8, ch. 29] has been considered in the control context, where the study focuses on how

This work was supported by the EU FP7 project RECONFIG, FP7-ICT600825, and Academy of Finland grant 286580. Co-funded by the Erasmus+ Programme of the European Union.

The authors are with the Intelligent Robotics Group, Department of Electrical Engineering and Automation, Aalto University, Finland. Email: \{Usama.tariq, rajkumar.muthusamy, Ville.kyrki\} daalto.fi

${ }^{1}$ Collaborative and cooperative words are used interchangeably in this paper. cooperative agents control the distribution of load while simultaneously handling a common object. However, the placements of grasps effect the load distribution significantly. Nevertheless, the load sharing has not been addressed from the perspective of planning cooperative grasps.

We propose a method for grasp coordination for multiple agents that allows coordinated grasping of unknown objects. The underlying idea of the method is to share the object load among the cooperative agents. To be specific, the method allows decentralized multi-agent grasp planning to determine cooperative grasps with minimal wrenches for a desired comanipulation task. We make two assumptions in our method (i) all candidate grasps are force closure (ii) each agent will have knowledge only about its own embodiment and capabilities. In conventional grasp planning, force closure is a minimal condition of a grasp to withstand arbitrary disturbances. For collaborative grasping in decentralized setting, force closure assumption eliminates the need of individual grasp analysis. Moreover, the decentralized agents are not able to predict the stability of grasp by other agents. Thus the assumption simplifies grasp planning. We represent the load sharing problem as a quadratic optimization problem to parametrize the constraint wrenches required for the task. Moreover, the formulation allows to tune the load sharing coefficient and define even the distribution of load in a flexible way (e.g. one of the agent can be set to assist or support or partner).

We also present a human-robot cooperative system which demonstrates a grasping for an unknown object consisting of human grasp detection, decentralized grasp planning, grasp execution and cooperative object manipulation. We formulate two experimental metrics to analyse cooperative grasp stability and load distribution. Experiments on physical robot demonstrate that the collaborative grasp solutions and their performance based on cost evaluation correlates with the experimental metrics. This validates the effectiveness of the proposed method in multi-agent systems.

In summary, the primary contributions of this paper are:

1) A grasp analysis approach that estimates the minimal wrenches satisfying the task criterion for each grasp pair and determines collaborative grasps for effective load distribution.

2) An online system for human-robot cooperation that allows grasp planning for unknown objects and enables to validate the effectiveness of the proposed methodology.

3) A study on performance of collaborative grasp solutions and two experimental measures to evaluate the quality of performed joint grasps. 
In the following, Sec. II surveys related work. Sec. III presents the grasp analysis methodology based on load sharing concept. The human-robot cooperative system that implements the method is described in Sec. IV. Sec. V studies the method in virtual and physical environment. Finally, Sec. VI concludes with future directions.

\section{RELATED WORKS}

Load sharing [8] has been studied as a control problem from two perspectives in multi-robot systems. On one side, several optimal load sharing strategies have been proposed to resolve actuation redundancies in co-manipulation tasks and allocate suitable wrench set-points for the co-manipulators. Zheng and Luh [9] proposed several distribution methods for two cooperating manipulators based on least energy consumption and minimal force consumption criterion. For unknown loads, load estimation and force compensation methods were presented in [10]. Recently in [11], dynamic distribution of load has been considered for heterogeneous cooperative manipulation setting, where a intuitive tuning strategy is presented to balance the payloads. On the other side, a few works presented load sharing methods for robust holding of a common object, where the objective is to determine cooperating robot wrenches needed for resisting external disturbances. Uchiyama and Yamashita [12] proposed adaptive load sharing by formulating the contact conditions as a set of linear inequalities with respect to load sharing coefficients. Later, they [13] defined control parameters and presented an algorithm using quadratic programming for adjusting exerted wrenches that allows to hold object robustly against the external disturbances. However, these strategies cannot be applied if a human is considered as a partner.

In human centred environments, the robot partner is capable of compensating the object dynamics and gravity in comanipulation tasks. Most works in the area consider role distribution between the robot and human and only few works have considered load sharing in this context. Lawitzky [14] presented an effort sharing strategy for joint manipulation tasks and three intuitive effort sharing policies for unilateral and balanced effort distributions. They used haptic interface to perform human-robot experiment in virtual environment. Nevertheless, in collaborative manipulation load sharing is also heavily dependent on relative locations of grasps.

Grasp planning [15], [16] has been studied extensively with individual robots and to an extent in multi-robot systems. Recently, multi-robot coordination has also been addressed in decentralized context with heterogeneous robots. The embodiment uncertainty was addressed by treating traditional grasp quality measures in a probabilistic context [17], [18]. However, the approaches are not applicable if no model for the manipulated is available in planning, as is the case in this paper. Task specific quality measures have also been studied extensively, primarily extending the wrench space formulation [19]. The approach proposed in this paper is inspired by the existing idea of formulating a task as required wrenches. However, for load sharing of unknown objects, we propose to estimate the task parameters using visual measurements of object shape. The method uses online measurements and quadratic programming to determine the grasp pair for an unknown object whereas the conventional grasp planning is more dependant on evaluation of grasps using wrench space quality measures for known objects.

\section{Grasp Planning For Collaborative MANIPULATION}

A grasp coordination method for effective load sharing is presented in this section to accomplish a collaborative manipulation task. For simplicity we consider a two-agent case where an intelligent first agent makes a grasp taking into account the existence of the other cooperative agent. Considering the grasp location of the first agent, this method selects a grasp for the second agent such that the load sharing objective function is maximized for desired task. Prior assumptions about the system are:

1) A sequential coordination protocol is used for collaboration between multi-agent systems.

2) Approximate grasp location of the first agent is known.

3) Approximate location of center of gravity of the object is known.

4) A number of candidate grasps are available for the second agent.

We show that these assumptions can be valid in Sec. IV.

\section{A. Task-specific wrench equilibrium}

During robotic manipulation of an object, a single grasp results in multiple contact points on the target object. In typical grasp stability analysis, each contact point is represented by a single force (frictionless contact) or a friction cone (contacts with friction) in the direction of surface normal at the contact point. The grasp will be stable (force closure) if any external wrench can be resisted by these contact forces. Therefore, a stable grasp will be applying forces and torques in one or multiple directions on the target object for execution of the manipulation task. Thus at any time instant during the manipulation, a stable grasp can be represented by a net wrench acting on the object.

$$
\boldsymbol{g}=\left[\begin{array}{l}
\boldsymbol{f} \\
\boldsymbol{\tau}
\end{array}\right]
$$

where $\boldsymbol{g}$ is the grasp wrench of an arbitrary stable grasp, $\boldsymbol{f}$ is the force vector, and $\tau$ is the torque vector acting on the target object in the object reference frame. The net wrench $\boldsymbol{g}$ on the object is the resultant of all forces and torques applied at the contact points by an $n$-fingered robot grasp [19].

Over the course of a manipulation task, the object undergoes a trajectory which can be discretized as a sequence of poses based on arbitrary time interval. At each pose during the manipulation, external wrenches such as gravitational force will be acting on the object. The manipulating agents need to cooperatively apply a wrench on the target object to compensate the external wrenches and change the object's state to next pose in the trajectory. In object reference frame, 
this wrench can be represented by a task wrench

$$
w_{t}=\left[\begin{array}{l}
f_{t} \\
\tau_{t}
\end{array}\right]
$$

where $f_{t}$ is the task force vector and $\tau_{t}$ is the task torque vector required in a particular pose. A similar approach for task representation has also been used by Seredyński [20] for task specific grasp planning.

Consider a target object being manipulated by two agents $A_{1}$ and $A_{2}$, with its center of gravity at $\boldsymbol{o}=[0,0,0]^{T}$ in object reference frame. Assume that the locations of grasps by $A_{1}$ and $A_{2}$ are represented by vectors $\boldsymbol{p}_{\mathbf{1}}=$ $\left[p_{1 x}, p_{1 y}, p_{1 z}\right]^{T}$ and $\boldsymbol{p}_{\mathbf{2}}=\left[p_{2 x}, p_{2 y}, p_{2 z}\right]^{T}$ respectively. Using the grasp representation discussed earlier, grasp wrenches by both agents can be expressed as $g_{1}=\left[\begin{array}{ll}f_{1} & \tau_{1}\end{array}\right]^{T}$ and $\boldsymbol{g}_{\mathbf{2}}=\left[\begin{array}{ll}\boldsymbol{f}_{\mathbf{2}} & \boldsymbol{\tau}_{\mathbf{2}}\end{array}\right]^{T}$.

To succeed in manipulation, these wrenches must be equal to the task wrench $\boldsymbol{w}_{\boldsymbol{t}}$. Thus the wrench equilibrium can be expressed as

$$
\begin{gathered}
f_{1}+f_{2}=f_{t} \\
p_{1} \times f_{1}+\tau_{1}+p_{2} \times f_{2}+\tau_{2}=\tau_{t}
\end{gathered}
$$

\section{B. Grasp optimization by minimization of efforts}

The utility of a $\boldsymbol{g}_{\mathbf{1}}-\boldsymbol{g}_{\mathbf{2}}$ grasp pair is proportional to the wrenches required from both agents for the manipulation task where smaller wrenches represent a better grasp solution. Therefore, for a particular pose during the manipulation, a cost function for a grasp pair can be defined as

$$
\begin{aligned}
c\left(\boldsymbol{g}_{\mathbf{1}}, \boldsymbol{g}_{\mathbf{2}}\right)= & \left\|\boldsymbol{f}_{\mathbf{1}}\right\|^{2}+\omega_{1}^{2}\left\|\boldsymbol{\tau}_{\mathbf{1}}\right\|^{2} \\
& +\epsilon^{2}\left\|\boldsymbol{f}_{\mathbf{2}}\right\|^{2}+\epsilon^{2} \omega_{2}{ }^{2}\left\|\boldsymbol{\tau}_{\mathbf{2}}\right\|^{2}
\end{aligned}
$$

The factors $\epsilon, \omega_{1}$, and $\omega_{2}$ are non-negative coefficients, introduced to incorporate capabilities of manipulating agents in cost factor. $\epsilon$ indicates the ratio between contribution desired from each agent, whereas $\omega_{i}$ is the factor of torque compared to force for $A_{i}$ in cost calculation. $\omega_{i}$ is typically related to the size of a gripper, which can be used as a scaling factor between forces and torques.

Setting $\epsilon=1$ represents equal desired efforts by both agents. In a lift-up task, such a solution will try to distribute the object's load equally between both agents. A value of less than 1 for $\epsilon$ will result in higher efforts by $A_{2}$ compared to $A_{1}$.

For a $\boldsymbol{g}_{\mathbf{1}}-\boldsymbol{g}_{2}$ grasp pair, the total cost of task execution can be calculated as sum of the costs over poses.

$$
c_{T}\left(\boldsymbol{g}_{\mathbf{1}}, \boldsymbol{g}_{\mathbf{2}}\right)=\sum_{i} c_{i}\left(\boldsymbol{g}_{\mathbf{1}}, \boldsymbol{g}_{\mathbf{2}}\right)
$$

where $c_{i}$ is the cost of $i$ th pose during the desired trajectory.

An optimum solution will offer a minimum total cost to ensure maximum quality. Since it was considered that the candidate grasps are available, the problem is reduced to grasp selection such that the cost of manipulation is minimized. Therefore, the load sharing problem can be considered as a quadratic minimization problem; minimizing cost expressed in (5) for each grasp pair while satisfying task equations presented in (3) and (4).
For the manipulation task, grasp wrenches are unknown for both grasps before task execution. However, minimum required wrenches for a known task wrench can be computed for each $\boldsymbol{g}_{\mathbf{1}}-\boldsymbol{g}_{\mathbf{2}}$ grasp pair where grasp by $A_{1}$ will remain same in all pairs. A candidate grasp by $A_{2}$ offering minimum total cost will result in an optimal collaboration under given cost factor. Quadratic programming [21] can be used to estimate minimum required wrenches.

The quadratic programming solution will estimate the unknown state vector $\boldsymbol{x}$ while minimizing the function

$$
\begin{gathered}
\qquad(\boldsymbol{x})=\frac{1}{2} \boldsymbol{x}^{T} Q \boldsymbol{x} \\
\text { s.t. } E \boldsymbol{x}+\boldsymbol{e}_{\mathbf{0}}=\mathbf{0}
\end{gathered}
$$

where

$$
x=\left[\begin{array}{l}
f_{1} \\
\tau_{1} \\
f_{2} \\
\tau_{2}
\end{array}\right]
$$

$$
\begin{gathered}
Q=\left[\begin{array}{cccc}
I & 0 & 0 & 0 \\
0 & \omega_{1}^{2} I & 0 & 0 \\
0 & 0 & \epsilon^{2} I & 0 \\
0 & 0 & 0 & \epsilon^{2} \omega_{2}^{2} I
\end{array}\right] \\
E=\left[\begin{array}{cccc}
I & 0 & I & 0 \\
R_{1} & I & R_{2} & I
\end{array}\right] \\
\boldsymbol{e}_{\mathbf{0}}=-\boldsymbol{W}_{\boldsymbol{t}} .
\end{gathered}
$$

The matrix $Q$ is derived from the cost factor expressed in (5). $I$ and 0 are $3 \times 3$ identity and zero matrices respectively. The matrix $R_{i}$ is a cross product operator given by

$$
R_{i}=\left[\begin{array}{ccc}
0 & -p_{i z} & p_{i y} \\
p_{i z} & 0 & -p_{i x} \\
-p_{i y} & p_{i x} & 0
\end{array}\right]
$$

such that the equality constraint $E \boldsymbol{x}+\boldsymbol{e}_{\mathbf{0}}=0$ is the equivalent representation of wrench equilibrium expressed in (3) and (4).

Estimated grasp wrenches can be used to calculate the cost of manipulation for a grasp pair using (5). For an established $A_{1}$ grasp at $\boldsymbol{p}_{\mathbf{1}}$, we determine grasp location of $A_{2}$ from a given set of grasp candidates $P_{2}=\left\{p_{2}^{1}, \ldots, p_{2}^{n}\right\}$ to accomplish a trajectory specified as a list of task wrenches $W_{t}=w_{t}^{1}, \ldots, w_{t}^{n}$ using

$$
p_{2}=\underset{p_{2}^{k} \in P_{2}}{\arg \min }\left(\sum_{i} \min _{x} c_{i}\left(x ; w_{t}^{i}, p_{1}, p_{2}^{k}\right)\right)
$$




\section{GRASPING SYSTEM FOR HUMAN-ROBOT \\ COLLABORATION WITH UNKNOWN OBJECTS}

An on-line grasping system has been developed for human-robot collaborative lift-up of unknown objects. A block diagram of the system is shown in Fig. 1. Depth information of the environment from a fixed Kinect is used as the input to the system. The depth input is filtered and segmented to extract the target from the environment. It is assumed that the object of interest is the largest object in the sensor's field of view and is placed on a planar surface.

The robotic grasp planning problem was divided between two planners. A pre-planner generates candidate grasps on the target object followed by a post-planner that selects an optimal candidate for collaborative manipulation. Components of the systems are briefly described in following subsections.

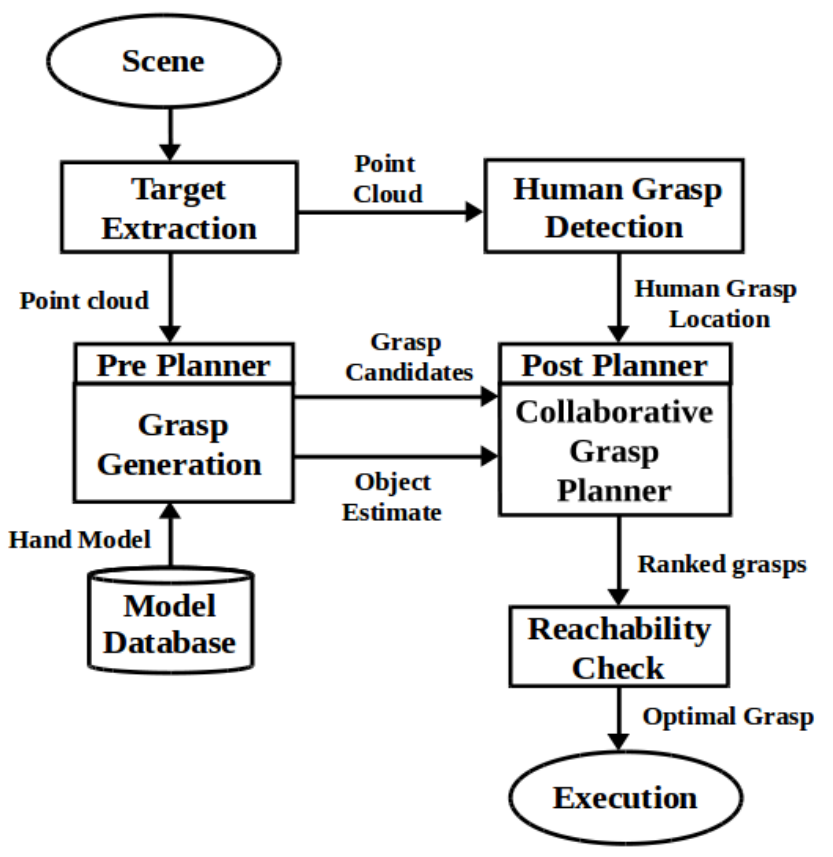

Fig. 1: Functional flowchart of a human robot collaboration system

\section{A. Target Extraction and Human Grasp Detection}

The object extraction process consists of filters applied in a preprocessing chain. First, a pass-through filter selects the area of interest in the sensor's field of view which is then down sampled using a voxel grid filter. The down sampling process reduces size of the point cloud while retaining shape of the target object. Subsequently, the ground plane is removed using RANSAC plane estimation. To remove random noise in the remaining point cloud, a statistical outlier filter is used. Since the target object is the largest object in the scene, the largest point cluster after Euclidean segmentation gives point cloud of the target object.

Point cloud of the target was used to detect location of the human grasp and to generate candidate grasps on the object. Grasp location was approximated by observing change in the target point cloud. A human grasp execution on the target introduces additional point cloud data close to the target object. The location was estimated by computing mean location of 100 points closest to the target object.

\section{B. Pre-planner: Grasp Generation}

The pre-planner is responsible for generating candidate grasps on the target object. An elementary grasp actions (EGA) [22] based method was used in this work for candidate grasp generation on unknown objects using a simplified gripper model. The partial point cloud of the target object was approximated with rectangles using plane estimation. EGA5 grasps [22] were generated on rectangular faces of the object while considering width of the gripper to filter graspable rectangular faces. Candidate grasps were validated for collision avoidance in the simulation environment to filter out invalid grasps. It was assumed that the candidate grasps generated by this method satisfy force closure.

Another primary parameter required for collaborative grasp analysis is center of gravity (CoG) of the object which was estimated by computing an axis aligned bounding box (AABB) of the object point cloud. The center of AABB was considered as the CoG. We assume that atleast two sides of the object are visible in the point cloud for $\mathrm{CoG}$ estimation using this method.

\section{Post-planner: Collaborative Grasp Planning}

The post planner evaluates the candidate grasps generated by pre-planner for collaborative manipulation task. A liftup of the target object was considered as the collaborative manipulation task with an assumption that accelerations during manipulation will be low such that the inertia of the object can be ignored. The task will thus include one goal state i.e. object in equilibrium after the lift-up. The task wrench can be expressed as $\boldsymbol{w}_{\boldsymbol{t}}=\left[\begin{array}{ll}\boldsymbol{f}_{\boldsymbol{g}} & \mathbf{0}\end{array}\right]^{T}$ where $\boldsymbol{f}_{\boldsymbol{g}}$ is the gravitational force. The task equations for the object after lift-up can be expressed as

$$
\begin{gathered}
f_{1}+f_{2}=f_{g} \\
p_{1} \times f_{1}+\tau_{1}+p_{2} \times f_{2}+\tau_{2}=0
\end{gathered}
$$

For a fixed human grasp, the cost of task execution was computed for each candidate grasp - human grasp pair using the method proposed in Sec. III and the candidates were ranked in ascending order of cost. A candidate grasp producing the minimum cost was selected for robotic grasp. Subsequently, the selected grasp was executed to complete the manipulation task.

It is to be noted that the gravitational force in the task wrench depends on mass of the object which cannot be estimated for an unknown object. However, a unity value can be assumed for the unknown mass since the optimal location for a robotic grasp will be independent of object's mass. A constant value of unknown mass used for cost evaluation of each candidate will scale the approximated cost with the same factor. 


\section{EXPERIMENTS AND RESULTS}

Experiments were performed to verify successful execution of collaborative manipulation task and validate load sharing between human and robot manipulator. A 7-DOF manipulator was used as the robotic agent with BarrettHand gripper. A stationary Kinect was used for sensing the environment. The manipulation task considered in experiments was a lift-up of $5 \mathrm{~cm}$ above the ground surface. A value of one was used for $\epsilon, \omega_{1}$ and $\omega_{2}$ in cost estimation. Thus the system will try to achieve an equal contribution by both agents in manipulation task. Such a parameter setting can be easily verified, quantitatively or by visual observation. To eliminate variations in a real human grasp execution between multiple experiments, the human grasp was emulated by a fixed support at the time of evaluating stability. This eliminates any bias in the results by ensuring an equal lift by both agents.

\section{A. Cooperative Grasp Stability}

A lift-up task requires translation of a target opposite to the direction of gravity and a stable cooperative solution should not change the orientation of the lifted object. Therefore, stability was evaluated by measuring tilt of the object after lift-up. Tilt was measured as the sum of absolute roll and pitch generated after the lift-up. The experiment was performed on two random poses for a table with a different human grasp location for each pose.

Figure 2a shows the table object in the first experimental setup along with a human grasp. Approximate location of human grasp, candidate grasp locations and robot grasp decision are shown in the visualization environment in Fig. $2 \mathrm{~b}$. To verify the quality of decision, the task was performed with six candidate robot grasps. The evaluated candidates are labeled (a-f) in Fig. $2 b$ and the state of the object after task execution is shown in Fig. 3.

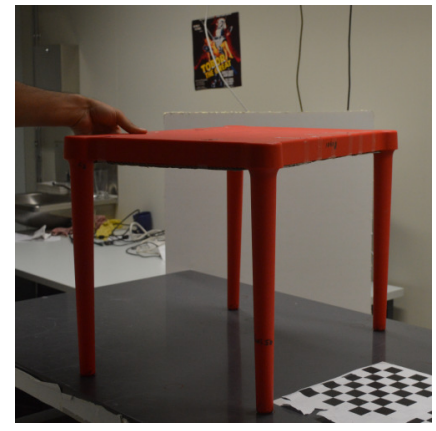

(a)

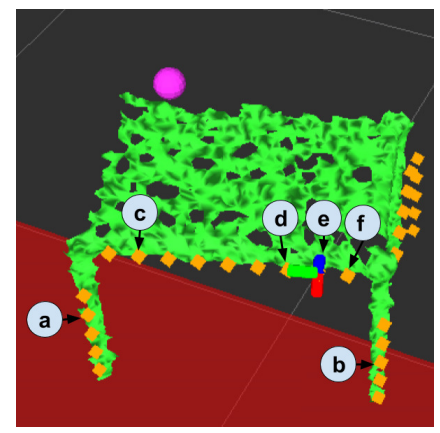

(b)
Fig. 2: Table object (pose 1) (a) Real environment with a human grasp (b) Visualization environment - Pink sphere is estimated location of human grasp, yellow squares are robot grasp candidates and rgb axes marker indicates grasp decision by proposed solution. Candidate grasps evaluated in this experiment are labeled (a-f).

It can be observed that the object tilted in all cases. The measured tilt angles for each grasp are shown in Fig. 4

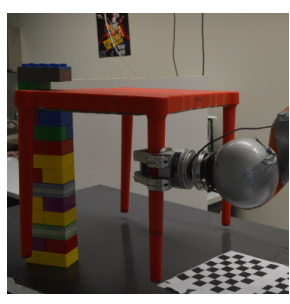

(a)

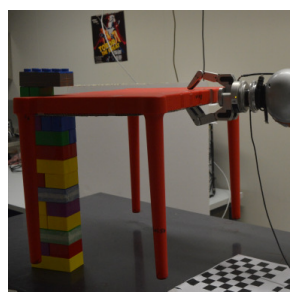

(d)

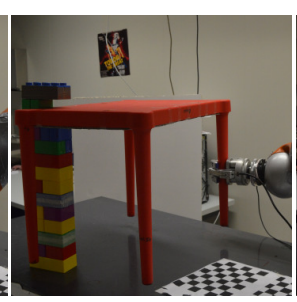

(b)

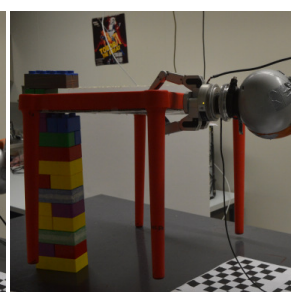

(c)

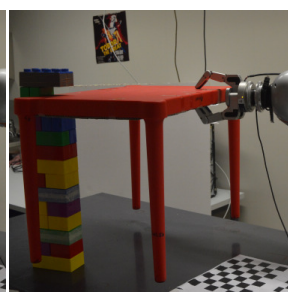

(e)

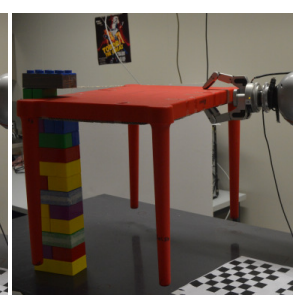

(f)
Fig. 3: Table object (pose 1) - 6 candidate robot grasps (a-f) executed against an emulated human grasp.

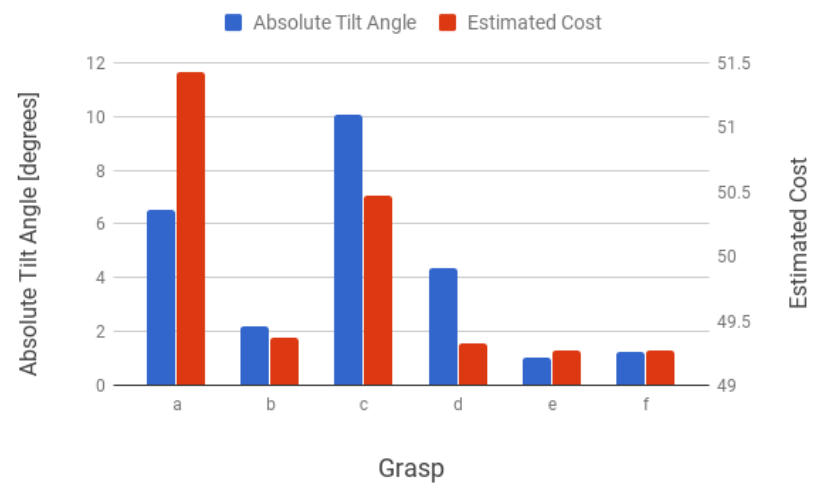

Fig. 4: Table object (pose 1) - Total absolute tilt angle and estimated cost for robot grasps (a-f).

along with the cost estimated prior to the task execution by proposed method. The tilt is minimum for Grasp (e), which was also selected for robot grasp by the proposed solution. This substantiates that the grasp analysis method selected the candidate grasp producing maximum stability in the object after task execution. Furthermore, it can be observed that the estimated cost factor correlates with the measured quality of task completion.

In Fig. 4, a noticeable irregularity is between the cost and quality measures of Grasp (a) and (c), where Grasp (a) estimated worse than Grasp (c) has shown a better stability. The difference is due to the difference in type of grasps evident in Fig. 3. In cost estimation, all candidate grasps were supposed to be equally stable which does not hold true in the real execution.

The same experiment was repeated on the table object with a different pose and human grasp location. Real and visualization environments are shown in Fig. 5 along with candidate grasp locations and selected robot grasp solution. 
Similar to the prior experiment, a lift of $5 \mathrm{~cm}$ was performed with pairs of fixed human grasp and six candidate robot grasps. Evaluated candidates are labeled (a-f) in Fig. 5b whereas Fig. 6 shows the state of table object after manipulation for each grasp pair.

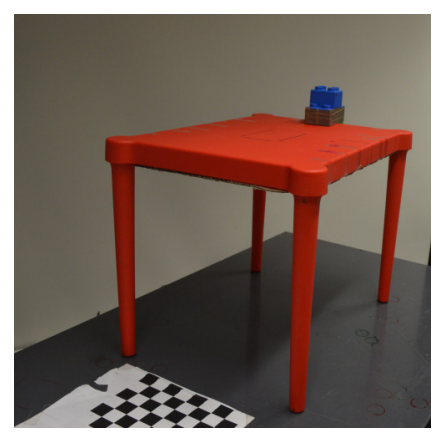

(a)

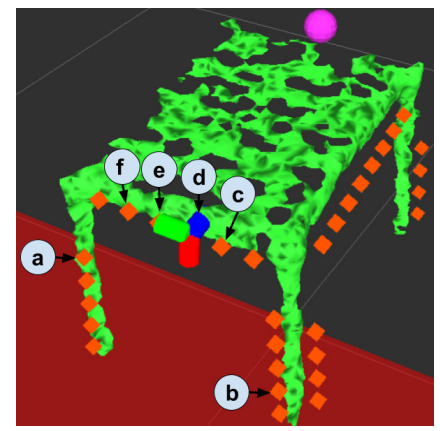

(b)
Fig. 5: Table object (pose 2) (a) Real environment with an emulated human grasp (b) Visualization environment Pink sphere is estimated location of human grasp, orange squares are robot grasp candidates and rgb axes marker indicates grasp decision by proposed solution. Candidate grasps evaluated in this experiment are labeled (a-f).

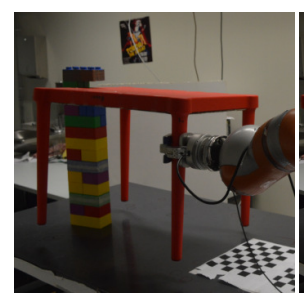

(a)

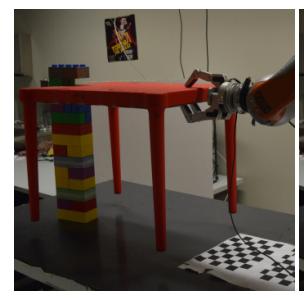

(d)

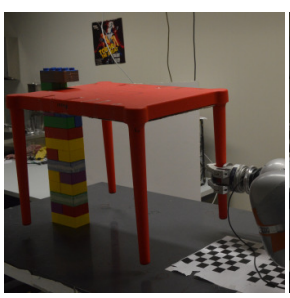

(b)

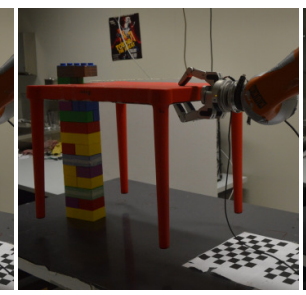

(e)

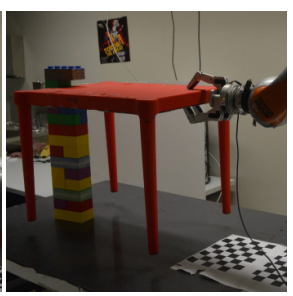

(c)

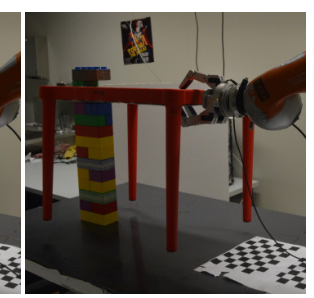

(f)
Fig. 6: Cooperative manipulation of the table (pose 2) - 6 candidate robot grasps (a-f) executed against an emulated human grasp.

Total tilt angles measured after the manipulation for each evaluated grasp pair are plotted in Fig. 7 along with the estimated cost. Grasp (d) was observed to have minimum tilt after manipulation which corresponds to the computed solution by the developed system. A correlation can again be observed between measured quality and estimated cost of the candidate grasps. An inconsistency can again be observed for Grasp (f) compared to the Grasp (a) or (b) due to the different types of grasps in real execution.

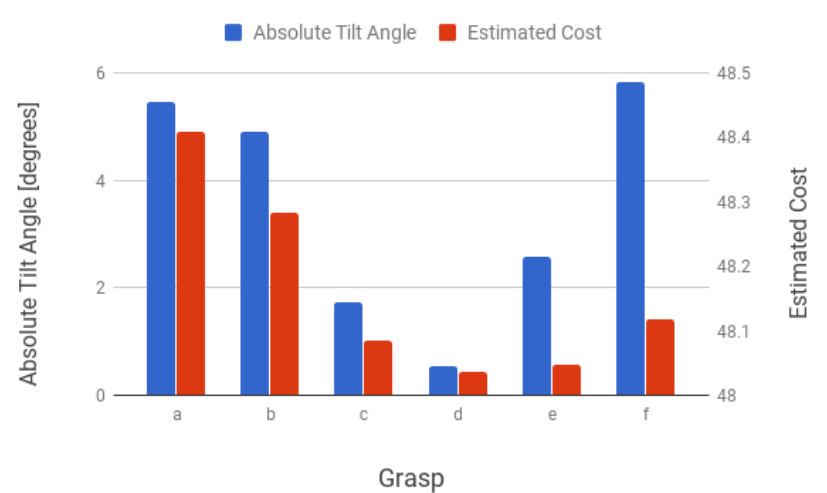

Fig. 7: Table object (pose 2) - Total absolute tilt angle and estimated cooperative manipulation cost for robot grasps (af).

The results indicate that stability defined using tilt correlates strongly with the minimization of exerted wrenches, allowing the proposed method to plan stable coordinated grasps. However, the capabilities of the hands appear nonsymmetric which could be further taken into account in the cost function by introducing different coefficients along different directions.

\section{B. Load Distribution}

The cost function used in the experiments aims to ensure load sharing between manipulating agents. Thus the robotic manipulator should bear half of the total load of the object. To verify that the grasp decision by the system is also an optimal load sharing solution, the manipulation task was performed on a box shaped object for multiple candidate grasp and a fixed human grasp location. The total load of the box, i.e. the gravitational force, was measured to be approximately $4.2 \mathrm{~N}$. Fig. 8a shows the experimental setup for this experiment. The task was repeated with six candidate grasps, labeled (a-f) in Fig. 8b. State of the object after task execution in each evaluated case is shown in Fig. 9.

After the lift-up task was completed, the force acting on the robotic gripper in direction of gravitation was measured using a force/torque sensor. The measured force for each evaluated candidate is plotted in Fig. 10. It can be observed that the load contribution by robot increases as it moves towards center of gravity of the object. An optimal load sharing solution must contribute half of the total load i.e. $2 \mathrm{~N}$ after the manipulation task, making Grasp (b) the optimal in this case. However, grasp decision by the proposed method was Grasp (a) which offers second closest value to the optimal $2 \mathrm{~N}$.

The difference in the decision is due to low mass of the object and the use of multi-fingered hand that may exert forces in different directions during grasping.

To validate that the load sharing is reflected in computed cost of manipulation, the estimated cost is plotted with the measured cost for all evaluated candidates in Fig. 11. The 


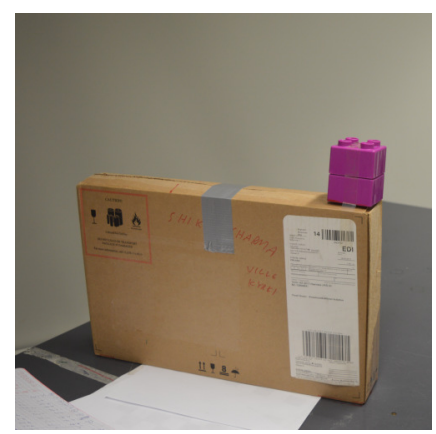

(a)

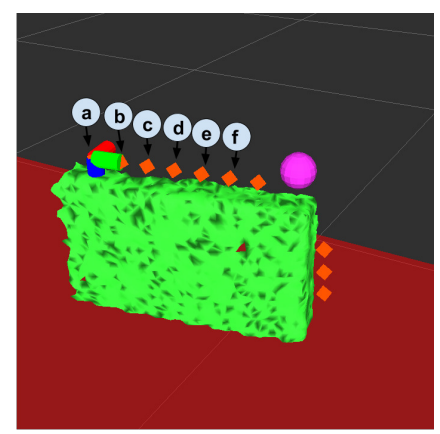

(b)

Fig. 8: Box object (a) Real environment with an emulated human grasp (b) Visualization environment - Pink sphere is estimated location of human grasp, orange squares are robot grasp candidates and rgb axes marker indicates grasp decision by proposed solution. Candidate grasps evaluated in this experiment are labeled (a-f).

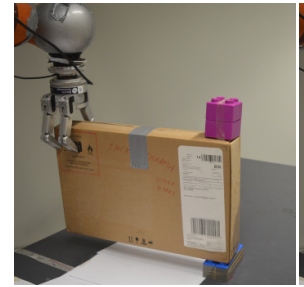

(a)

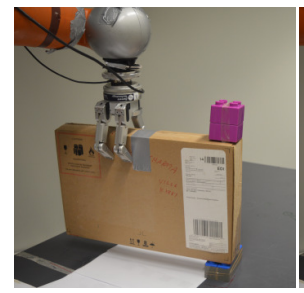

(d)

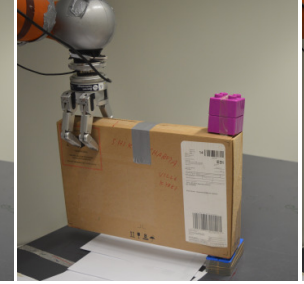

(b)

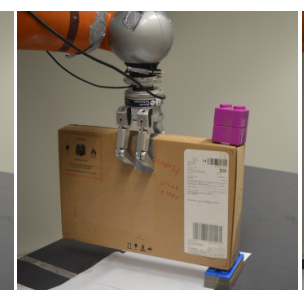

(e)

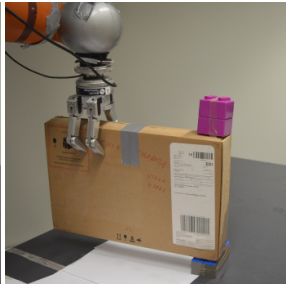

(c)

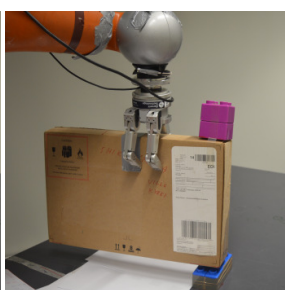

(f)

Fig. 9: Cooperative manipulation of box object - 6 different robot grasps executed against an emulated human grasp.

measured cost is computed using the expression:

$$
c_{m}=\left\|\boldsymbol{f}_{\boldsymbol{m}}\right\|^{2}+\left(\left\|\boldsymbol{f}_{\boldsymbol{g}}\right\|-\left\|\boldsymbol{f}_{\boldsymbol{m}}\right\|\right)^{2}
$$

where $f_{g}$ is the gravitational force on the object and $f_{m}$ is the measured force for each grasp plotted in Fig. 10. An increasing trend can be observed in both measured and estimated costs indicating a correlation between both factors. However, increase in the measured cost is not as gradual as in estimated cost factor. This is due to the fact that the measured cost does not include the torque factors. Furthermore, due to dynamics of the experimental setup, the object was completely lifted by robot for Grasp (e) and (f) which further increased forces on the gripper. Similarly, the emulated grasp having multiple points of contact with the target had different effects for different grasps.

The force measurements indicate that the proposed method

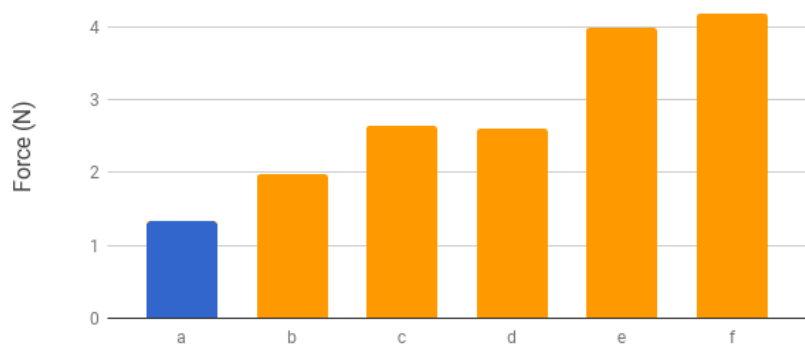

Grasp

Fig. 10: Box object - Forces experienced by gripper for Grasps (a-f). Grasp (a) is the grasp decision by proposed solution.

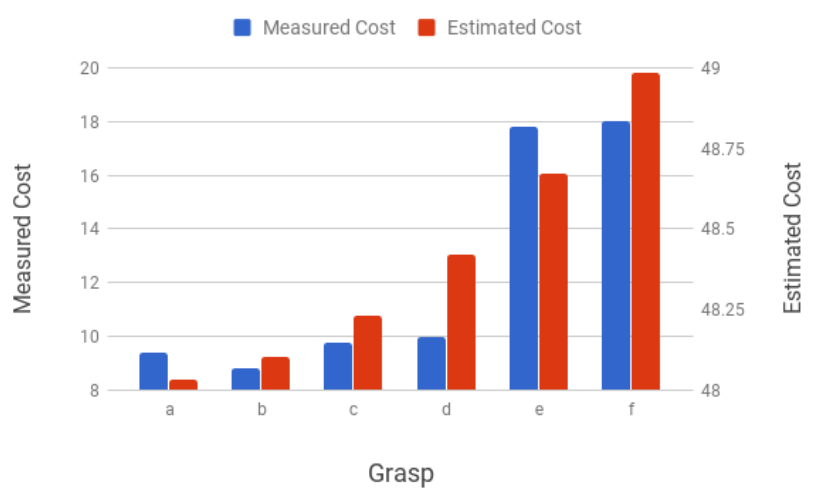

Fig. 11: Box object - Measured and estimated cost for grasps (a-f).

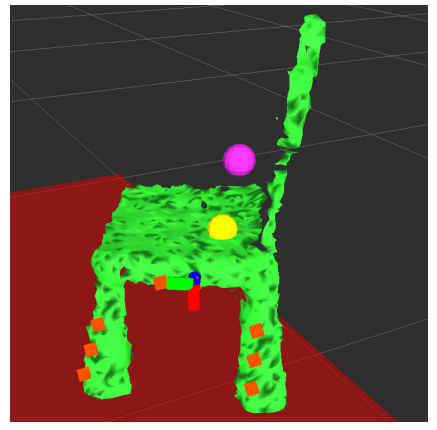

(a)

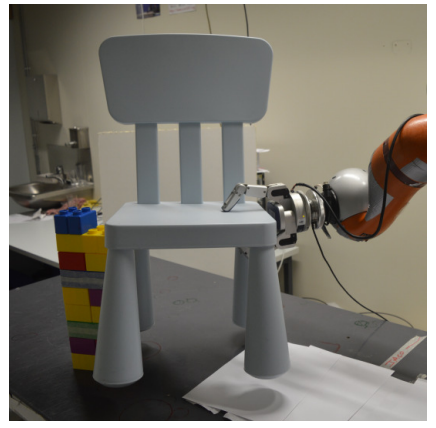

(b)
Fig. 12: Cooperative manipulation with a chair object. (a) Visualization environment - The point cloud acquired from a single Kinect view is visualized as a green triangulated mesh, pink sphere is estimated location of human grasp, yellow sphere is the estimated location of center of gravity, orange squares are candidate robot grasp locations and rgb axes marker indicated grasp decision. (b) Cooperative lift-up by selected solution.

can be used for load distribution among agents. However, the 
quality of load distribution is dependent on the accuracy of estimated parameters such as location of the object center of gravity.

\section{Collaborative manipulation of complex shaped objects}

To verify robustness of the system, the manipulation task was repeated with complex shaped objects. It was observed that if a close approximation of object's center of gravity and human grasp location can be extracted, successful cooperative manipulation can be achieved by the system. Figure 12 shows the manipulation task being performed on a chair object.

\section{CONCLUSION}

This paper proposed a grasp coordination method for optimal load sharing in collaborative manipulation. The approach aims to determine collaborative grasps that maximize the distribution of load among the agents, differing from conventional grasp planning. We presented a human robot collaborative system to experimentally demonstrate and study the performance of the proposed methods. In order to validate the effectiveness of the load sharing grasp solutions for physical systems, we developed metrics to quantify the quality of the executed collaborative grasps. Experimental results showed that the collaborative grasp solutions and their performance based on cost evaluation correlate with the experimental metrics. The method showed potential for collaborative manipulation in human centric environments.

Solution quality of the method can be improved by using better grasp planers for generating grasp candidates. In this work, we assumed the grasp candidates to be force closure for the case of unknown objects. Better approaches to quantify the candidate grasps of such object can add value to our method. Considering sensitivity of the method to center of gravity, a better CoG estimator can also improve results for complex objects. The task considered in this work was a lifting task but the method formulation is not limited to such tasks. The performance in more complex tasks should be evaluated in future work.

\section{REFERENCES}

[1] A. Edsinger and C. C. Kemp, "Manipulation in human environments," in Humanoid Robots, 2006 6th IEEE-RAS International Conference on. IEEE, 2006, pp. 102-109.

[2] C. Smith, Y. Karayiannidis, L. Nalpantidis, X. Gratal, P. Qi, D. V. Dimarogonas, and D. Kragic, "Dual arm manipulationa survey," Robotics and Autonomous systems, vol. 60, no. 10, pp. 1340-1353, 2012.

[3] Z. Yan, N. Jouandeau, and A. A. Cherif, "A survey and analysis of multi-robot coordination," Int. J. Adv. Robot. Sys., vol. 10, pp. 1-18, 2013.
[4] A. Farinelli, L. Iocchi, and D. Nardi, "Multirobot systems: A classification focused on coordination," IEEE Trans. Syst., Man, Cybern. B, vol. 34, no. 5, pp. 2015-2028, 2004.

[5] T. Arai, E. Pagello, and L. E. Parker, "Guest editorial advances in multirobot systems," IEEE Trans. on Robot. Autom., vol. 18, no. 5, pp. 655-661, Oct 2002.

[6] L. E. Parker, "Distributed intelligence: Overview of the field and its application in multi-robot systems," Journal of Physical Agents, vol. 2, no. 1 , pp. $5-14,2008$.

[7] C. C. Kemp, A. Edsinger, and E. Torres-Jara, "Challenges for robot manipulation in human environments [grand challenges of robotics]," IEEE Robotics \& Automation Magazine, vol. 14, no. 1, pp. 20-29, 2007.

[8] B. Siciliano and O. Khatib, Springer Handbook of Robotics. Secaucus, NJ, USA: Springer-Verlag New York, Inc., 2007.

[9] Y. F. Zheng and J. Y. S. Luh, "Optimal load distribution for two industrial robots handling a single object," in Proceedings. 1988 IEEE International Conference on Robotics and Automation, 1988, pp. 344349 vol.1.

[10] K. I. Kim and Y. F. Zheng, "Unknown load distribution of two industrial robots," in Proceedings. 1991 IEEE International Conference on Robotics and Automation, Apr 1991, pp. 992-997 vol.2.

[11] A. Z. Bais, S. Erhart, L. Zaccarian, and S. Hirche, "Dynamic load distribution in cooperative manipulation tasks," in Intelligent Robots and Systems (IROS), 2015 IEEE/RSJ International Conference on. IEEE, 2015, pp. 2380-2385.

[12] M. Uchiyama and T. Yamashita, "Adaptive load sharing for hybrid controlled two cooperative manipulators," in Proceedings. 1991 IEEE International Conference on Robotics and Automation, Apr 1991, pp. 986-991 vol.2.

[13] M. Uchiyama and Y. Kanamori, "Quadratic programming for dexterous dual-arm manipulation," in Int.Symp.on Robotics, Mechatronics and Manufacturing Systems, 1993, pp. 367-372.

[14] M. Lawitzky, A. Mrtl, and S. Hirche, "Load sharing in human-robot cooperative manipulation," in 19th International Symposium in Robot and Human Interactive Communication, Sept 2010, pp. 185-191.

[15] J. Bohg, A. Morales, T. Asfour, and D. Kragic, "Data-driven grasp synthesis;a survey," IEEE Transactions on Robotics, vol. 30, no. 2, pp. 289-309, April 2014.

[16] A. Sahbani, S. El-Khoury, and P. Bidaud, "'an overview of 3d object grasp synthesis algorithms"," "Robotics and Autonomous Systems", vol. 60, no. 3, pp. 326 - 336, 2012, autonomous Grasping. [Online]. Available: http://www.sciencedirect.com/science/article/pii/S0921889011001485

[17] R. Muthusamy and V. Kyrki, "Decentralized approaches for cooperative grasp planning," in Int. Conf. Contr. Autom. Robot. Vis. (ICARCV), Dec 2014, pp. 693-698.

[18] R. Muthusamy, C. P. Bechlioulis, K. J. Kyriakopoulos, and V. Kyrki, "Task specific cooperative grasp planning for decentralized multi-robot systems," in IEEE Int. Conf. Robot. Autom., May 2015, pp. 6066-6073.

[19] M. A. Roa and R. Suárez, "Grasp quality measures: review and performance," Autonomous Robots, vol. 38, no. 1, pp. 65-88, 2015. [Online]. Available: http://dx.doi.org/10.1007/s10514-014-9402-3

[20] D. Seredyński, T. Winiarski, K. Banachowicz, and C. Zieliński, "Grasp planning taking into account the external wrenches acting on the grasped object," in Robot Motion and Control (RoMoCo), 2015 10th International Workshop on. IEEE, 2015, pp. 40-45.

[21] D. Goldfarb and A. Idnani, "A numerically stable dual method for solving strictly convex quadratic programs," Mathematical programming, vol. 27, no. 1, pp. 1-33, 1983.

[22] D. Aarno, J. Sommerfeld, D. Kragic, N. Pugeault, S. Kalkan, F. Wörgötter, D. Kraft, and N. Krüger, "Early reactive grasping with second order 3d feature relations," in Recent Progress in Robotics: Viable Robotic Service to Human. Springer, 2007, pp. 91-105. 